\title{
Simulation Research of Extension Control Based on Crane-Double Pendulum System
}

\author{
Gang Yang(Corresponding Author), Weiwei Zhang \& Ying Huang \\ Automation College, Guangdong University of Technology \\ 729E.Dongfeng Rd. Guangzhou 510090, China \\ E-mail: yanggang@china.com.cn \\ Yongquan $\mathrm{Yu}$ \\ Computer College \\ Guangdong University of Technology \\ 729E.Dongfeng Rd. Guangzhou 510090, China
}

The research is financed by National Natural Science Foundation of China (60272089) and Guangdong Provincial Natural Science Foundation of China (04009464)

\begin{abstract}
According to the existed structure and algorithm of extension controller, proposed an improved extension control algorithm based on the Optimal Control, which was named LQR-EC, and applied to a SIMO system-Crane-Double Pendulum System. And, using MATLAB simulation platform to study the effect of the LQR-EC Algorithm. The result shows that, the LQR-EC Algorithm not only has a simple theory, but also has rapid response and stability.
\end{abstract}

Keywords: Extension Control, Crane-Double Pendulum System, Simulation Research

\section{Introduction}

Extension Control, a concept and method of intelligent control, was established and developed on the basis of Extension Theory, which was originated from China. In the recent years, many experts and scholars have carried out the corresponding research of the structure and algorithm of Extension Controller, and have achieved certain results.

Crane-Double Pendulum System is a non-linear, multi-variables and complex system. It is an ideal model of testing various control theory. In this paper, for crane-double system proposed LQR-EC Algorithm, which is based on the existing EC Algorithm and Optimal Control Theory. The simulation research shows that the LQR-EC Algorithm can satisfy the purpose of improving the control effect, and steadying the system more quickly.

\section{Proposed of Extension Control Method}

In 1991, Wang Shine-yu, a Professor of East China University of Science and Technology, proposed a new concept and method of Intelligent Control, denominated Extension Control, which basic idea is to solve control problem form the aspect of information transformation. In other word, it is to transform the control information into the eligible value range by using dependent degree to tune the output modification factors. He is the opener of Extension Control. Based on this idea, they set up the structure of Extension Controller is shown in Fig.1.

Fig. 1 shows the structure of extension controller, which adopts dual-layer structure including upper-layer extension controller and basic extension controller.

The basic extension controller consists of five parts: character selecting, character pattern identification, dependent degree identification, measure pattern identification and control algorithm. The basic extension controller is used for completing the main control function.

The upper-layer extension controller consists of database, repository, and manage of decision-making and information. Database and repository are used for saving the information of control process and expert knowledge respectively. As 
the basic extension controller's consummate and supplement, this upper-layer controller is used to optimize the basic control, guarantee the good control effect, and reflect the transform of contradictory question, which is the extension control emphasized.

The extension control theory and method has provided the basis theory and method for people to solve the existent contradictory questions in the automatic control system such as stability, accuracy and speed.

In 1996, Pan Dong and Jin Yi-hui proposed a more complete structure of extension control, and improved the control algorithm. All of these enabled the extension controller can use a simple knowledge to self-study, consequently to achieve the better control effect. In 2005, Huang Ying and Yu Yong-quan proposed an improved extension control algorithm by adopting dependent degree and status distance, the basic extension controller's whole output of algorithm is as follows:

$u(t)=\left\{\begin{array}{cc}u(t-1), & K(s)>=0 \\ y(t) / k-p \cdot K(s) \cdot \operatorname{sgn}(e)+D(s) \cdot \operatorname{sgn}(e) \cdot g, & -1 \leq K(s)<0 \\ u_{m}, & K(s)<-1\end{array}\right.$

Where, $u(t)$ is the current output value, $u(t-1)$ is the previous control value, $y(t)$ is the current sampling value of control object, $k$ is the static gain, $K(s)$ is the dependent degree of character status, $D(s)$ is the status distance, $p$ is modification factor, $\operatorname{sgn}(e)$ is the symbol function of error $e$ shown in the follows:

$\operatorname{sgn}(e)=\left\{\begin{array}{cc}1, & e>0 \\ 0, & e=0 \\ -1, & e<0\end{array}\right.$

\section{Modeled and analyzed of Crane-Double Pendulum System}

In the actual production, it requests the crane to deliver the goods to the destination as soon as possible, and does not allow to present wide range of swinging in the process of moving. This requests the crane to maintain the two angles' steady, moreover the car itself must achieve the destination at last. All of these will be achieved through motor by the control of controller. The model of Crane-Double System is as shown in Fig.2.

Where, $M$ is the crane's equivalent quality, $m_{1}$ is the upper-pendulum's equivalent quality, $m_{2}$ is the lower-pendulum's equivalent quality, $x$ is the car's position, $\alpha$ is the angle $1, \quad \beta$ is the angle $2, l_{1}$ is the length of the upper-pendulum, $l_{2}$ is the length of the lower-pendulum, $F$ is the pulling force of the car received.

Analyzed the model of crane-double pendulum system, and adopted $x=[x, \dot{x}, \alpha, \dot{\alpha}, \beta, \dot{\beta}]^{\prime}$ as the system's feature vector. The vector expresses the car's position, car's response speed, the upper-pendulum's angle, the upper-pendulum's response angular speed, the lower-pendulum's angle, and the lower-pendulum's response angular speed respectively.

And from the analysis, we know that $x=\dot{x}=\alpha=\dot{\alpha}=\beta=\dot{\beta}=0$ is the system's balance point. First, doing linear processing of the system nearby the balance point, and then taking the actual parameters of crane-double pendulum system are as follows: motor's load $J=1 \times 10^{-4} \mathrm{~kg} \cdot \mathrm{m}^{2}$, anti-electromotive force coefficient $\mathrm{Ke}=0.4758 \mathrm{~V} \cdot \mathrm{s}$, armature resistance $R a=13.5 \Omega$, moment coefficient $K t=0.0491 \mathrm{~kg} \cdot \mathrm{m} / \mathrm{A}$, transmission round radius $r=0.02276 \mathrm{~m}, m_{1}=0.3 \mathrm{~kg}, m_{2}=0.5 \mathrm{~kg}$, $M=0.4 \mathrm{~kg}, l_{1}=0.205 \mathrm{~m}, l_{2}=0.156 \mathrm{~m}$.

Finally, we can obtain the state-space equation of the crane-double pendulum system is:

$\left\{\begin{array}{l}\dot{x}=A x+B u \\ y=C x+D u\end{array}\right.$

Where, $A=\left[\begin{array}{ccclcc}0 & 1 & 0 & 0 & 0 & 0 \\ 0 & -58.1558 & -13.3099 & 0 & 0 & 0 \\ 0 & 0 & 0 & 0 & 0 & 0 \\ 0 & -73.7445 & -112.7311 & 0 & 79.6748 & 0 \\ 0 & 0 & 0 & 0 & 0 & 1 \\ 0 & 73.7445 & 112.7311 & 0 & -247.1962 & 0\end{array}\right], \quad B=\left[\begin{array}{c}0 \\ 9.4888 \\ 0 \\ 46.275 \\ 0 \\ -46.275\end{array}\right], \quad C=$ eye $(6), \quad D=0$.

With the aid of the MATLAB software, analyzed the performance of system's mathematical model, we find that this crane-double pendulum system is able to be controlled and observed completely.

\section{LQR-EC Algorithm}

The crane-double pendulum system is a SIMO system, the input is a voltage value, which indicates the car's target location, 6 outputs correspond to the 6 condition quantities of the state space equation. 
For this single-input and multi-outputs system, adopts error $(e)$ and error differential $(\dot{e})$ as the characteristic quantity of extension control, which are shown as follows:

$e=\sum_{i=1}^{3} k_{i} e_{i}, \dot{e}=\sum_{i=1}^{3} k_{i} \dot{e}_{i}$

Where, $e_{i}$ and $\dot{e}_{i}$ are the error and error differential which correspond the response of car's position, the upper-pendulum's angle and the lower-pendulum's angle respectively; $k_{i}$ is the weighted factor, and its value range is $0<k_{i}<1$.

Assumed that the allowable value ranges of weighted error $(e)$ and weighted error differential $(\dot{e})$ of control object are $\left[-e_{i o m}, e_{i o m}\right]$ and $\left[-\dot{e}_{i o m}, \dot{e}_{i o m}\right]$ respectively, the maximum extensible value ranges of weighted error $(e)$ and weighted error differential $(\dot{e})$ of control object are $\left[-e_{i m}, e_{i m}\right]$ and $\left[-\dot{e}_{i m}, \dot{e}_{i m}\right]$ respectively. So the extension set about character status $S(e, \dot{e})$ of basic extension controller can be represented in Fig.3.

In Fig.3, assumed that character status of the original point is $S_{0}(0,0)$, and point $S(e, \dot{e})$ is a character status of a random point in the coordinate plane.

Here defines that the distance from a random point of the coordinate plane to the original point of the coordinate plane is called status distance, written as $D_{S}$.

Then from Fig. 3 we can get the maximum status distance of classic domain and extension domain, together with the status distance of a random point of the coordinate plane can be represented by the following equations:

$D_{0}=\sqrt{e_{i o m}{ }^{2}+\dot{e}_{i o m}{ }^{2}}, D_{m}=\sqrt{e_{i m}{ }^{2}+\dot{e}_{i m}{ }^{2}}, D_{S}=\sqrt{e_{i}{ }^{2}+\dot{e}_{i}^{2}}$

Now defines that $K(s)=\left\{\begin{array}{cc}1-\left|S S_{0}\right| / M_{0}, & S €[\text { Classic-domain }] \\ \left(M_{0}-\left|S S_{0}\right|\right) /\left(M_{-1}-M_{0}\right), & S \notin[\text { Classic-domain }]\end{array}\right.$ is the character status

dependent degree of a random point $S(e, \dot{e})$ in the coordinate plane. Where $\left|S S_{0}\right|=\sqrt{K_{1} e_{i}{ }^{2}+K_{2} \dot{e}_{i}{ }^{2}}$, and $K_{1}$ and $K_{2}$ are the modification factors which were decided by the character pattern of themselves.

The dependent degree $K(s)$ indicates the connection degree of character status $S(e, \dot{e})$ and extension set. And the measure pattern of system can be divided from analysis of $K(s)$.

For the extension controller mainly works in the scope of system's unstable and critical-stable stage, and the LQR algorithm has already could realize the preliminary control of the crane-double pendulum system, so output value of the improved LQR-EC algorithm of basic extension controller can be given as follows:

(1) Measure pattern $M_{1}$

The character status of measure pattern $M_{1}$ is in the classic domain, which is able to be controlled completely, written as $M_{1}=\{s \mid K(s) \geq 0\}$. So, we adopt linear quadratic optimal control algorithm in this measure pattern. The output of controller is:

$u(t)=-K x$

Where $K=\left[K_{1}, K_{2}, \ldots, K_{n}\right]$ is the feedback gain matrix obtained from the control of LQR, $x$ is the feature vector of crane-double pendulum system.

(2)Measure pattern $M_{2}$

The character status of measure pattern $M_{2}$ is in the extension domain, written as $M_{1}=\{s \mid K(s) \geq 0\}$. So, we adopt the improved extension control algorithm in this measure pattern, named LQR-EC algorithm. The output of controller is:

$u(t)=-K x-K_{c i} \cdot K(s) \cdot \operatorname{sgn}(e)+D(s) \cdot g \cdot \operatorname{sgn}(e)$

Where $K(s)$ is the dependent degree of character status $S ; K_{C i}$ is the control parameter of the measure pattern $M_{2 i}$; $D(s)$ is the status distance; $g$ is the modification factor, $e$ is the weighted error; $\operatorname{sgn}(e)$ is the symbol function or weighted error $e$. 
(3)Measure pattern $M_{3}$

The character status of measure pattern $M_{3}$ is in the no-domain, written as $M_{3}=\{s \mid K(s)<-1\}$, the output of controller adopts maximum value. The output of controller is:

$u(t)=u_{m}$

From the above analysis, the whole LQR-EC algorithm of basic extension controller is as follows:

$u(t)=\left\{\begin{array}{cc}-K x, & K(s)>=0 \\ -K x-K_{c i} \cdot K(s) \cdot \operatorname{sgn}(e)+D(s) \cdot g \cdot \operatorname{sgn}(e), & -1 \leq K(s)<0 \\ u_{m}, & K(s)<-1\end{array}\right.$

\section{Simulation research of extension control}

For the model of crane-double pendulum system which obtained from the second part, we using the linear feedback extension controller to simulate the system model by the help of the simulation tool of MATLAB.

The controller is designed to control the pendulums to swing when there is a step-input for the system, and the pendulums can return to the upright position finally. And at the same time, the car can arrive at the new target location. The LQR-EC algorithm is programmed by the S-function of MATLAB, with one output and seven inputs (the first six inputs are the motion states of system, and the seventh input is the car's target location).

The original state of system is $x(0)=0, \dot{x}(0)=0, \alpha(0)=1.2, \dot{\alpha}(0)=0, \beta(0)=-1.2, \dot{\beta}(0)=0$. And the angles of pendulums are expressed with the Arc measure. The target location of the car is $x=0.2$; the expectation state is $x(0)=0.2, \dot{x}(0)=0, \alpha(0)=0, \dot{\alpha}(0)=0, \beta(0)=0, \dot{\beta}(0)=0$.

The result of simulation research of the car and pendulums are shown in Fig4.

\section{Conclusion}

From the shown of Fig.4, we found that compared with LQR Algorithm, the LQR-EC Algorithm mainly works in the system of unstable and critical-stable stage. The improved extension control algorithm not only can achieve the ideal control effect, but the more importantly is that it can reduce the system's control time and cause the system to stabilize quickly.

\section{References}

Huang Ying, Yu Yongquan, Zhang Ling, “Extension Control Algorithm Using Extension Set In Control System”[C]. Proceedings of the third International Conference on Information Technology and Applications(ICITA'05), 2005.

Liu Jinkun, “Advanced PID Control and MATLAB Simulation”[M]. Beijing: Publishing House of Electronics Industry, 2004.

Pan Dong and Jin Yihui, “Exploration and Research on Extension Control'[J]. Control Theory and Applications, 1996.

Shi Li and Liu Wei, “Design of Extension Controller for Mobile Robot”[J].Micro Computer Information, 2008, 4-2: 224-225.

Wang Shienyu and Li Jian, “Extension Control’[J].Control Theory and Applications, 1994.

Yang Chunyan, Cai Wen, “Extension Engineering” [M]. Beijing: Scientific Publishing House, 2007.

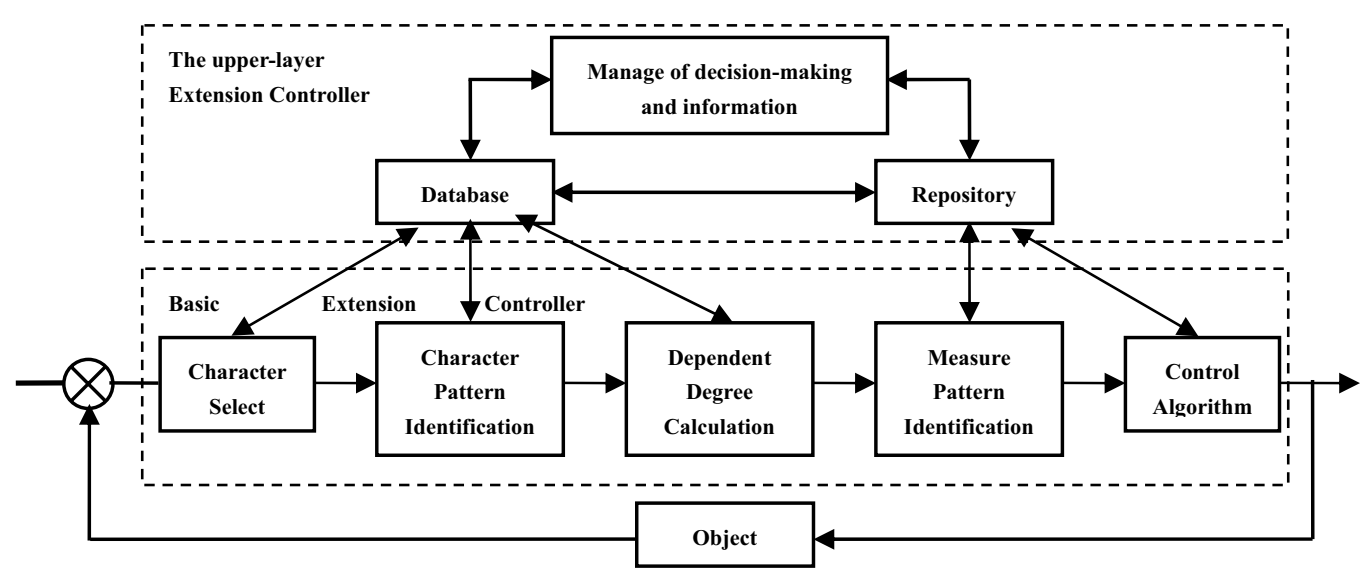

Figure 1. The structure of Extension Controller 


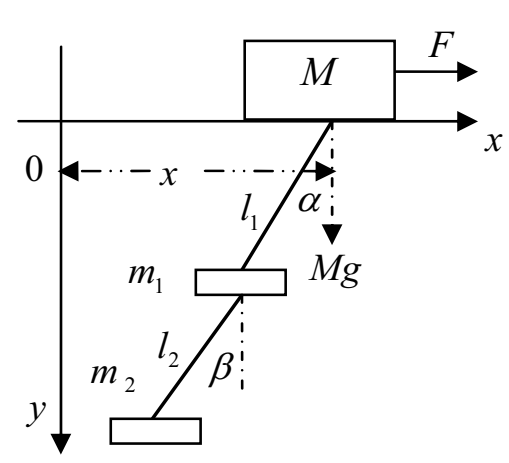

Figure 2. Model of Crane-Double System

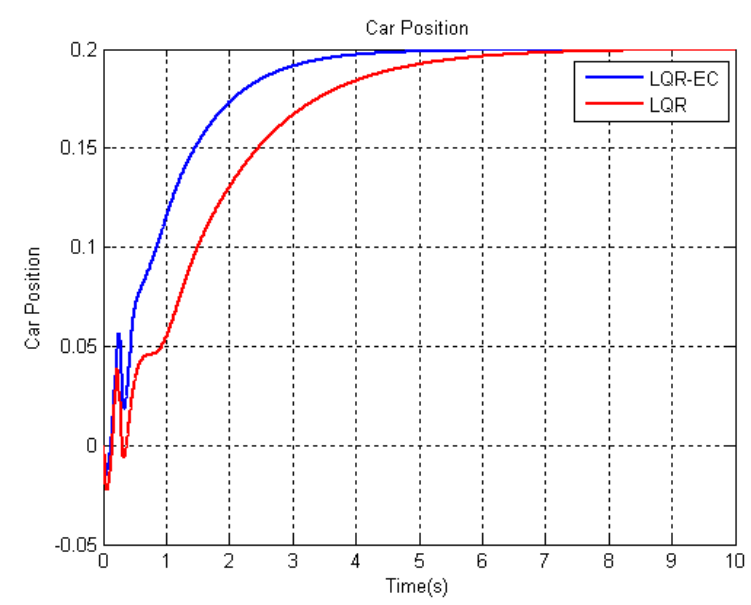

(1) response of car's position

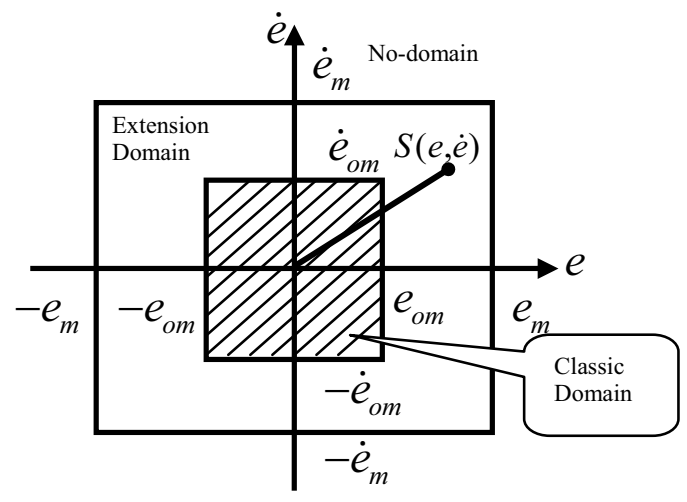

Figure 3. The extension set about character status $S(e, \dot{e})$

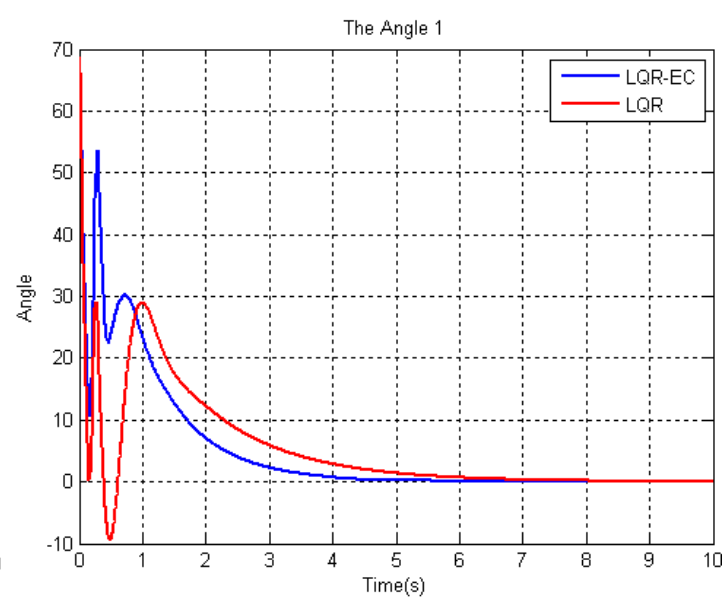

(2) response of the upper-pendulum's angle

Figure 4. Response of the system 